\title{
Review Article \\ Nanoparticle Imaging with Polarization Interferometric Nonlinear Confocal Microscope
}

\author{
Kohei Fujita and Chikara Egami \\ Faculty of Engineering, Shizuoka University, 3-5-1 Johoku, Naka-ku, Hamamatsu 432-8561, Japan \\ Correspondence should be addressed to Kohei Fujita; f0330133@ipc.shizuoka.ac.jp \\ Received 8 January 2014; Revised 1 April 2014; Accepted 2 April 2014; Published 27 April 2014 \\ Academic Editor: Kai Sun
}

Copyright (C) 2014 K. Fujita and C. Egami. This is an open access article distributed under the Creative Commons Attribution License, which permits unrestricted use, distribution, and reproduction in any medium, provided the original work is properly cited.

Polarization interferometric nonlinear confocal microscope has been developed for single nanoparticle analysis of drug delivery system (DDS). The microscope is a system based on a low cost and low power continuous wave (CW) laser light source. Also, the microscope observed shape anisotropy of the $200 \mathrm{~nm}$ diameter nanoparticle. According to nanoparticle imaging and CTF (contrast transfer function) curve observation of the microscope, three-dimensional resolution of the microscope measured up to $10 \mathrm{~nm}$.

\section{Introduction}

Nanoparticles have attracted tremendous attention because of microscopic properties, which can be exploited in many optical and medical applications [1-4]. Recently, particular attention, in our laboratory, has been devoted to an application to drug delivery system (DDS) [5]. An important thing to note is that the size and pharmacological properties of a single nanoparticle in matrix are key factors for DDS use. With a measurement of particles less than a half wavelength of an incident wave, however, general optical microscopes have poor spatial resolving power without toxic fluorescent indicator probes.

In this paper, we propose polarization interferometric nonlinear optical confocal microscope, which is a confocal microscope having high three-dimensional resolution even with continuous wave (CW) laser, incorporating a polarization Michelson interferometer. As with Raman microscope and CARS microscope, the microscope proposed observes the dielectric medium under the diffraction limit without fluorescent probes [6].

We verified this hypothesis by means of CTF (contrast transfer function) measurement and performed threedimensional measurement of a single nanoparticle.

\section{Materials and Methods}

2.1. Theoretical Model. The polarization interferometric nonlinear confocal microscope proposed enhances CTF of images. In short, the enhancement of CTF is an increase in the absolute value of the subtraction between the maximum and the minimum value of a spatial confocal signal. The techniques for realizing this goal are mentioned below.

One of the techniques enhancing CTF is an increase in the maximum value of a spatial confocal signal distribution, generating third-order nonlinear scattered light from the microscopic region of a dielectric medium.

Assume that focused coherent light is incident on a sample, where a small absorptive semihomogeneous objective region is in the background of a homogeneous transparent one. Matching the center spot of the airy disk generated by focused beams onto a region of semihomogeneous medium, high intense incident light induces third-order nonlinear polarization $\mathbf{P}^{(3)}$. The light intensity of the spot is much higher than the one of the region. Therefore, a scattered nonlinear signal $\mathbf{E}_{\mathrm{SN}}$ from the spot becomes noticeable. The effect raises the maximum of a scattered signal.

The other one of the techniques enhancing CTF is a decrease in the minimum value of a spatial confocal signal 
distribution, suppressing the background electric field $\mathbf{E}_{\mathrm{SL}}$ with a polarization interferometer. General confocal microscopes detect not only nonlinear scattered light depending on the intensity near a focal point but also linear scattered light. The polarization interferometer employed sets the subtraction between two electric fields, $\mathbf{E}_{R}$ and $\mathbf{E}_{\mathrm{SL}}$, to zero ideally, where $\mathbf{E}_{R}$ is the $y$-polarized field vector of a reference beam. As a result, a scattered signal from a region except for semihomogeneous regions becomes minimum value.

The following is to verify the process of setting $\mathbf{E}_{\mathrm{SL}}$ to zero theoretically. Firstly, suppose that two coherent beams of light interfere on a photodetector. In accordance with the principle of superposition, an interferometric electric field vector $\mathbf{E}_{o}$, at a point on the photodetector, is given by

$$
\mathbf{E}_{o}=\mathbf{E}_{S}+\mathbf{E}_{R}
$$

where $\mathbf{E}_{S}$ is the elliptically polarized field vector of the scattered light. The two fields are traveling in the $z$-direction. Written in Johns matrix column form, they are

$$
\begin{gathered}
\mathbf{E}_{S}=A_{S}\left(\begin{array}{c}
\cos \Psi \\
\sin \Psi e^{i \delta}
\end{array}\right), \\
\mathbf{E}_{R}=A_{R}\left(\begin{array}{l}
0 \\
1
\end{array}\right) .
\end{gathered}
$$

Here, $A_{S}$ and $A_{R}$ are the field amplitudes of the two vectors, respectively. $\Psi$ is the angle between principal axes of ellipse and the $(x, y)$-coordinate axes, and $\delta$ is the phase difference between the $x$ and $y$ components of $\mathbf{E}_{S}$. Secondly, suppose then that we introduce an analyzer in front of the detector, whose transmission axis makes a counterclockwise angle $\theta$ to be positive with the coordinate system. The rotated Johns matrix for the analyzer has the mathematical form

$$
[M(\theta)]=\left[\begin{array}{cc}
\cos ^{2} \theta & \sin \theta \cos \theta \\
\sin \theta \cos \theta & \sin ^{2} \theta
\end{array}\right] .
$$

As a result, the interferometric electric field vector through the analyzer and a pinhole is given by

$$
\begin{aligned}
\mathbf{E}_{o} & =[M(\theta)]\left(\mathbf{E}_{S}+\mathbf{E}_{R}\right) \\
& =\left[\begin{array}{l}
A_{S} \cos ^{2} \theta \cos \Psi+\sin \theta \cos \theta\left(A_{S} \sin \Psi e^{i \delta}+A_{R}\right) \\
A_{S} \sin \theta \cos \theta \cos \Psi+\sin ^{2} \theta\left(A_{S} \sin \Psi e^{i \delta}+A_{R}\right)
\end{array}\right] \\
& =\left[\begin{array}{l}
E_{x} \\
E_{y}
\end{array}\right] .
\end{aligned}
$$

We now consider the case where a high intense laser beam with $x$-polarization illuminates a sample. In addition, both field amplitudes are approximately equal, $A_{S} \cong A_{R}$, in this

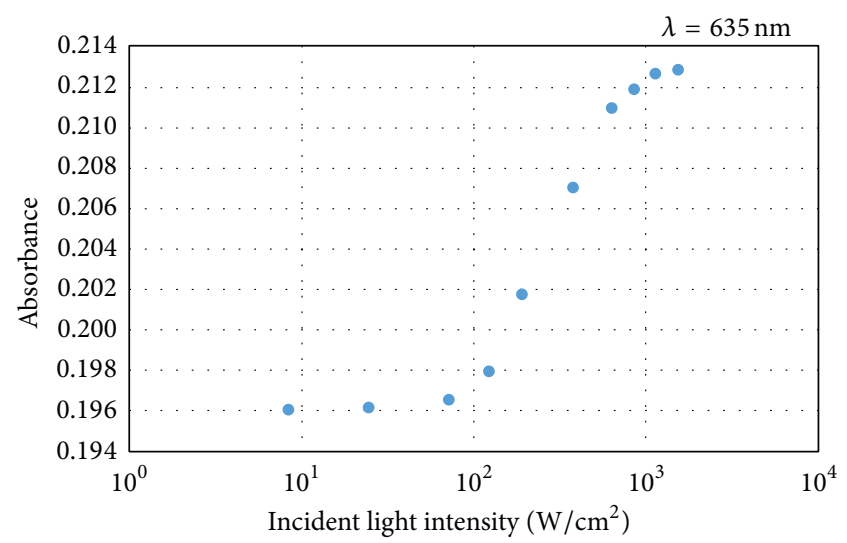

FIGURE 1: Nonlinear optical characteristic of the absorbance depending on the incident light intensity.

case. The scattered light intensity through the analyzer with its transmission axis at $\theta=-\pi / 4$ is, according to (4), given by

$$
\begin{aligned}
\mid \mathbf{E}_{o}(\theta & \left.=-\frac{\pi}{4}\right)\left.\right|^{2} \\
& =\left|\mathbf{E}_{x}\left(\theta=-\frac{\pi}{4}\right)\right|^{2}+\left|\mathbf{E}_{y}\left(\theta=-\frac{\pi}{4}\right)\right|^{2} \\
& =\left|A_{S}\right|^{2}\left(1-\cos \Psi+\sin \Psi \cos \delta-\frac{1}{2} \sin 2 \Psi \cos \delta\right) .
\end{aligned}
$$

In this case, (5) shows a function of the polarization interferometer subtracting the electric field vectors of the scattered light and the reflected reference beam. No subtraction signal of $\left|\mathbf{E}_{o}(\theta=-\pi / 4)\right|^{2}$ emerged from the semihomogeneous background region. To set the subtraction between the $x$ and $y$ components of the interference light to zero, we adjust the transmission axis at $\theta=-\pi / 4$. Consequently, the contrast resolution of the confocal image of a sample is enhanced; that is, CTF comes close to infinity ideally.

2.2. Sample Preparation. In our research, we employed an inhomogeneously chromophore-doped polystyrene particle (200 $\mathrm{nm}$ diameter) as a sample. According to the preceding section, this sample is a small absorptive semihomogeneous medium in the background of a homogeneous transparent one. Figure 1 shows an absorbance characteristic of nanoparticles for incident light intensity. A thin sample $\left(\alpha_{0} L=\right.$ 0.45 ) was used in this measurement, where $\alpha_{0}$ is absorption coefficient and $L$ is the thickness of a sample.

The polarization interferometric nonlinear confocal microscope proposed is aiming to be used for observing a medium in a single nanoparticle for DDS. The nanoparticle with spatial anisotropic structure usually has no inversion symmetry. On the one hand, for a macroscopic region of interest, it has anisotropy structure. On the other hand, for a microscopic region of interest, it is a semihomogeneous region. The third-order nonlinear scattered light depending on the microstructure in a homogeneous region is generated, though its intensity is very low. Based on this measurement, 


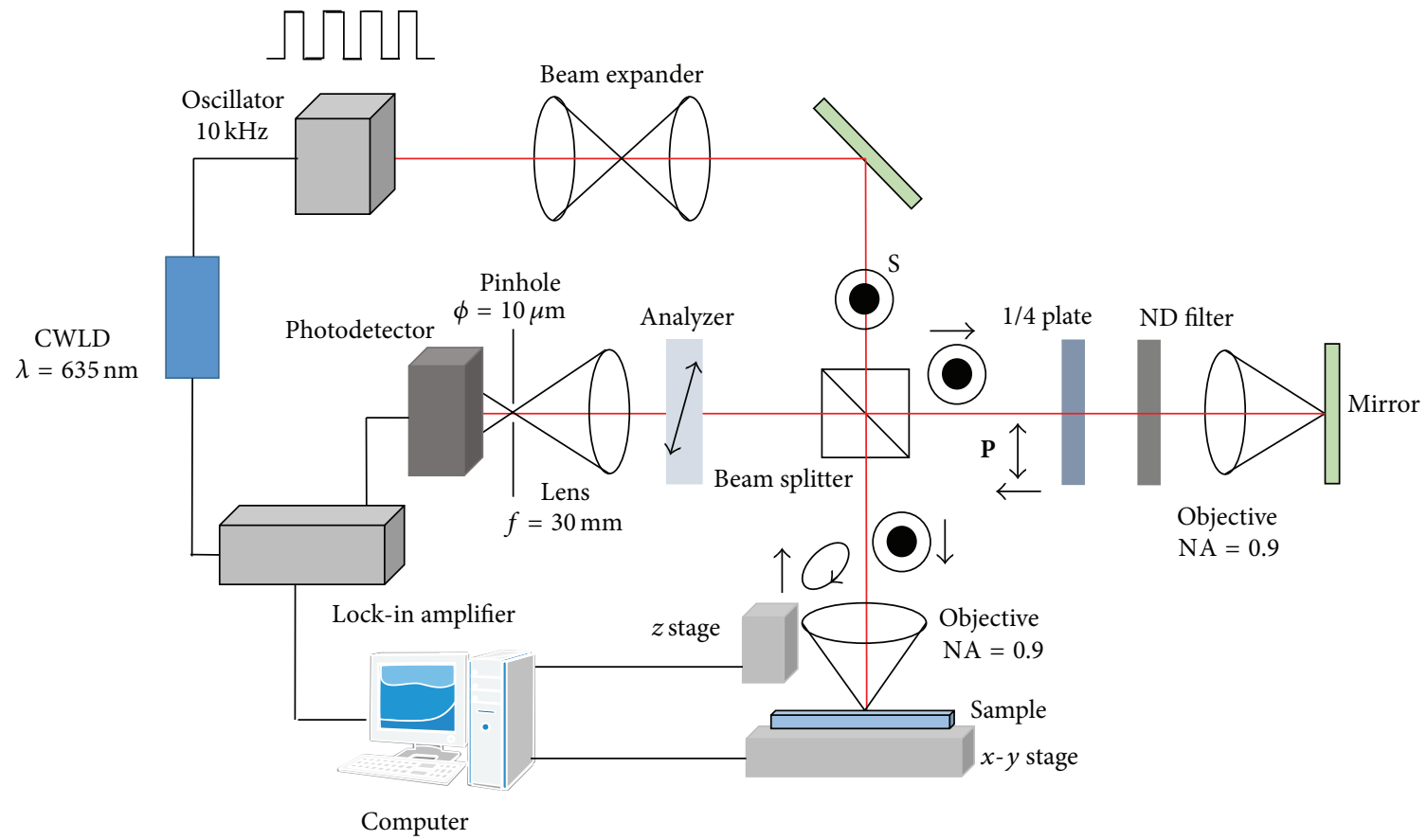

FIGURE 2: Optical setup for a polarization interferometric nonlinear confocal microscope.

an image of the microstructure in a single nanoparticle is produced without doping fluorescent probes.

\subsection{Experimental Setup. Figure 2 shows a schematic of a po-} larization interferometric nonlinear confocal microscope. This microscope is a confocal microscope including the Michelson interferometer for polarization interferometry. In consideration of optical spectrum of chromophore doped into nanoparticles inhomogeneously, a CW laser diode (LD) with an emission wavelength of $\lambda=635 \mathrm{~nm}$ is employed.

A LD's output beam as being S-polarized travels through a beam expander to optimize the numerical aperture (NA) of objective lenses, which determines the basic resolving power of the confocal microscope. The beam divides into reference-arm and measurement-arm beams. The referencearm and measurement-arm beams are focused onto a mirror and a sample, respectively, by two identical microscope objective lenses with a NA of 0.9. On the reference-arm, a quarter-wave plate is arranged. On the measurement-arm, on the other hand, a sample is mounted on $1 \mathrm{~nm}$ resolution three axis Piezo Stages. The measurement beam focused onto the sample induces a nonlinear dielectric polarization $\left(\mathbf{P}^{(3)}\right)$, which provides backscattering from it. Analysis of the scattered signal estimates the dielectric polarization on the construction of the sample. Assume that an optical axis of the medium has an orientation that makes an angle $\theta$, but not being perpendicular or parallel to the polarization vector orientation of the focused beam. The analyzer is initially positioned with its transmission axis parallel to a polarization vector orientation $(\theta=0)$ of the focused beam. In this case, the microscope functions as the conventional confocal one.
In addition, the condition that the focused beam's polarization and analyzer are completely crossed $(\theta=\pi / 2)$ prevents the vertical component from passing through the analyzer. After passing through a $10 \mu \mathrm{m}$ diameter pinhole, the interferometer's output intensities into a lock-in amplifier are measured. The current modulated LD for improving the signalto-noise ratio in the interferometer is employed to generate a chopping frequency of $10 \mathrm{kHz}$ for the lock-in reference.

We measured the axial resolution of the fundamental confocal microscope by using a mirror as a sample. The full width at half maximum (FWHM) is defined as the microscope's resolution. The basic confocal microscope has an axial resolution of $0.7 \mu \mathrm{m}$, which is limited by its NA, approximately being equal to theoretical value.

2.4. Measurement Procedure. In our experiment, the microscope mainly functions as the polarization-type interferometer to measure a chromophore distribution and, in turn, an optically anisotropic distribution in a nanoparticle [7]. The measurement procedure is as follows. Firstly, beams are focused on a background region in which there is no chromophore. Here, reference light's power is so adjusted that a polarization interference signal is minimized to enhance the CTF of signal light. After that, the laser beam is made to move to a region in which there is chromophore. Shown as the theoretical equation (4), a polarization interference signal is generated in response to the fractional change in $\Psi$ and $\theta$. This three-dimensional signal scan gives a chromophore distribution in a single nanoparticle. This results in a bright 


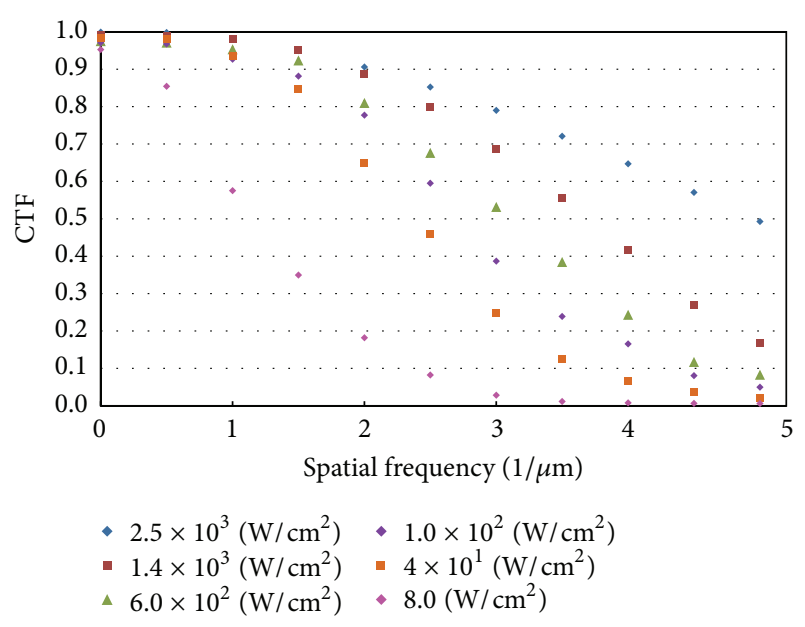

FIGURE 3: CTF curves measured with polarization interferometric nonlinear confocal microscope.

sample image on a darker background. The semihomogeneous areas with $\mathbf{P}^{(3)}$ can be observed in the microscope [8].

\section{Results and Discussion}

Following experiments determines how the polarization interferometric nonlinear confocal microscope proposed has three-dimensional resolution for observing a single nanoparticle.

In the first place, we measured CTF of confocal signals along a straight line passing through center points of two nanoparticles, which are separated by a certain distance, with the microscope proposed. A maximum value and minimum one of a scattered signal give a contrast resolution. Figure 3 shows CTF curves at six different levels of incident light intensities. These six different levels we selected are under light intensities not to induce photobleaching. Here, spatial frequency is the reciprocal of the distance between centers of two nanoparticles. According to Figure 3, the contrast resolution measured at the incident light intensity $(I=2.5 \times$ $\left.10^{3} \mathrm{~W} / \mathrm{cm}^{2}\right)$ is the highest of six different levels. In other words, the in-plane resolution depends on the intensity of incident light. An increase in incident light intensity induces a number of the third-order nonlinear polarization $\mathbf{P}^{(3)}$ from this measurement result. The system has realized high contrast measurement.

In the second place, the microscope proposed produces tomographic images in a plane, perpendicular to the axis $(z$ axis), including the focus of converged beams. Focusing beams on any point in a medium and scanning on the plane, a tomographic image like Figure 4 is constructed. Assume that the top surface of a nanoparticle is zero in the optical axis, which is set to $z$-axis direction. Figure 4 shows tomographic images at $z=100 \mathrm{~nm}$, whose scanning area is $200 \mathrm{~nm} \times$ $200 \mathrm{~nm}$. We got tomographic images at every $10 \mathrm{~nm}$ from $z=$ $0 \mathrm{~nm}$ to $z=200 \mathrm{~nm}$. The internal chromophore distribution becomes clear with increasing incident light intensity.
In the final place, Figure 5 shows a result of three-dimensional measurement. Figure 5(a) is a three-dimensional image of a single nanoparticle, measured with general confocal microscope at the incident light intensity $(I=1.0 \mathrm{~W} /$ $\mathrm{cm}^{2}$ ). This result means that general confocal microscope reveals surface shape of a single nanoparticle, though it is unsuitable for observing internal chromophore distribution. In short, the resolution of this microscope is not enough to observe a submicroarea in a nanoparticle. Furthermore, Figures 5(b), 5(c), and 5(d) show three-dimensional anisotropic distribution in a single nanoparticle, measured with the microscope proposed. The third-order nonlinear polarization $\mathbf{P}^{(3)}$ is not induced by the incident light, whose representative value of the intensity is $I=8.0 \mathrm{~W} / \mathrm{cm}^{2}$ in the linear region (see Figure 5(b)). This prevents obtaining fine chromophore distribution in a single nanoparticle. On the other hand, in the nonlinear region, the higher the incident light intensity is, the clearer the chromophore distribution is.

Here, the nonlinear region is classified into two regions, nonlinear moderate intensity region (see Figure 5(c)) and nonlinear high intensity region (see Figure 5(d)), whose representative values of the incident light intensity are $I=1.0 \times$ $10^{2} \mathrm{~W} / \mathrm{cm}^{2}$ and $I=2.5 \times 10^{3} \mathrm{~W} / \mathrm{cm}^{2}$, respectively. According to Figures 5(c) and 5(d), the distribution of chromophore being scattered for the incident light intensity in the nonlinear high intensity region is more precise than for the one in the nonlinear moderate intensity region.

In other words, clear $\mathbf{P}^{(3)}$ detection by making the incident light intensity high gives the chromophore distribution of a submicroarea of interest in a single nanoparticle. As a result, the contrast resolution of the microscope depends on the incident light intensity.

In our experiment, absolute coordinates are not important to obtain relative coordinates for a displacement of chromophore in a single nanoparticle and to measure an exact shape of an object. Zero point correction is not performed in repeated scan operations for this reason in Figure 5.

According to a tomographic image of a single nanoparticle in the nonlinear high intensity region, this incident light intensity increases the resolution of the in-plane direction up to $10 \mathrm{~nm}$. Therefore, Figure 5(d) is a three-dimensional image produced from the measurement with the microscope, whose three-dimensional resolution is $10 \mathrm{~nm}$.

\section{Conclusions}

Polarization interferometric nonlinear confocal microscope has been invented for future use of nanoparticle in DDS. The microscope is composed of a confocal microscope and a polarization interferometer. These two components enhance the axial resolution and the in-plane resolution, respectively. We confirmed the in-plane resolution of the microscope, depending on the incident light intensity, by measuring CTF curves. Also, we have succeeded in observing a threedimensional distribution in a chromophore-doped single nanoparticle (200 $\mathrm{nm}$ diameter). 


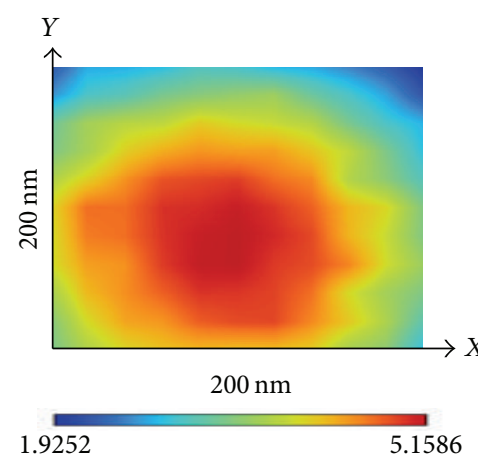

(a)

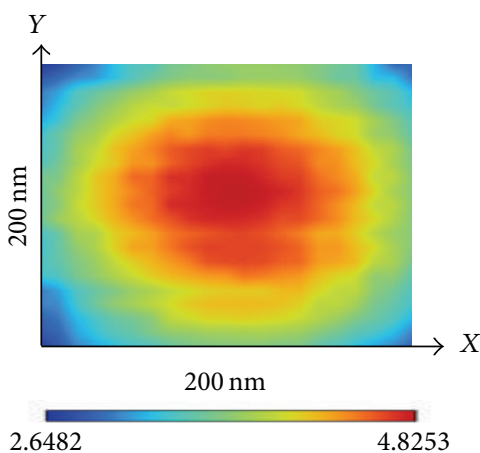

(b)

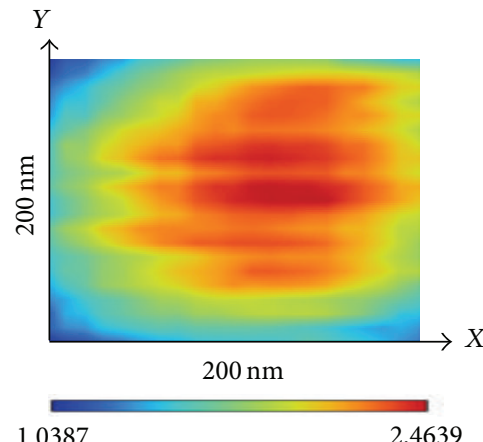

(c)

FIGURE 4: Tomographic images of a nanoparticle $(z=100 \mathrm{~nm})$, measured with polarization interferometric nonlinear confocal microscope: (a) linear region $\left(I=8.0 \mathrm{~W} / \mathrm{cm}^{2}\right)$, (b) nonlinear moderate intensity region $\left(I=1.0 \times 10^{2} \mathrm{~W} / \mathrm{cm}^{2}\right)$, and (c) nonlinear high intensity region $\left(I=2.5 \times 10^{3} \mathrm{~W} / \mathrm{cm}^{2}\right)$.

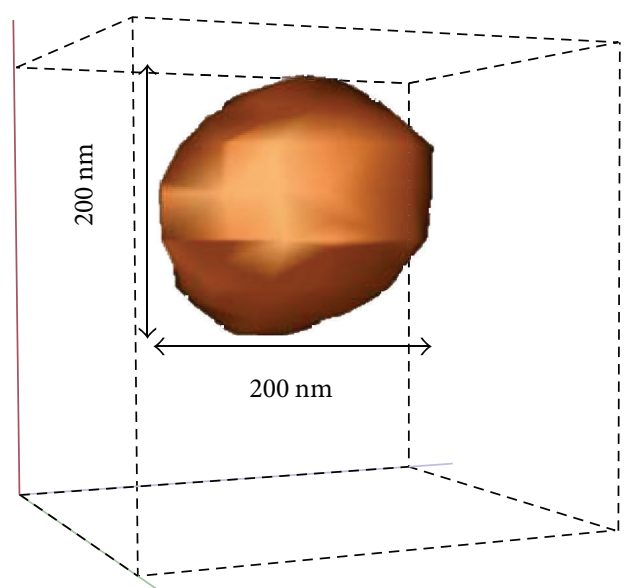

(a)

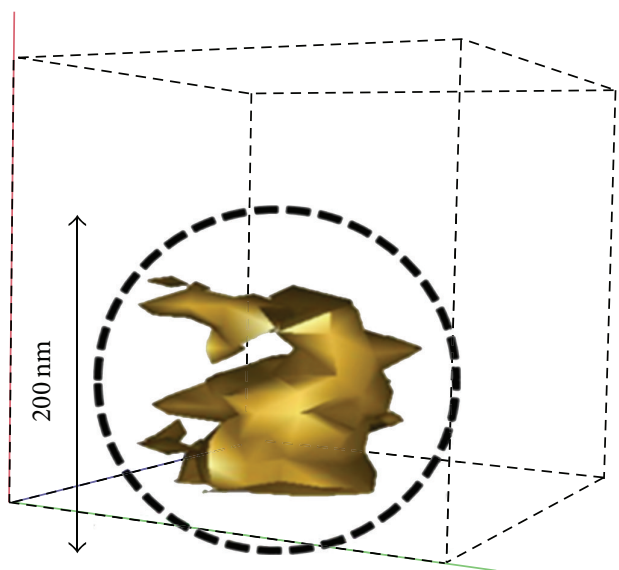

(c)

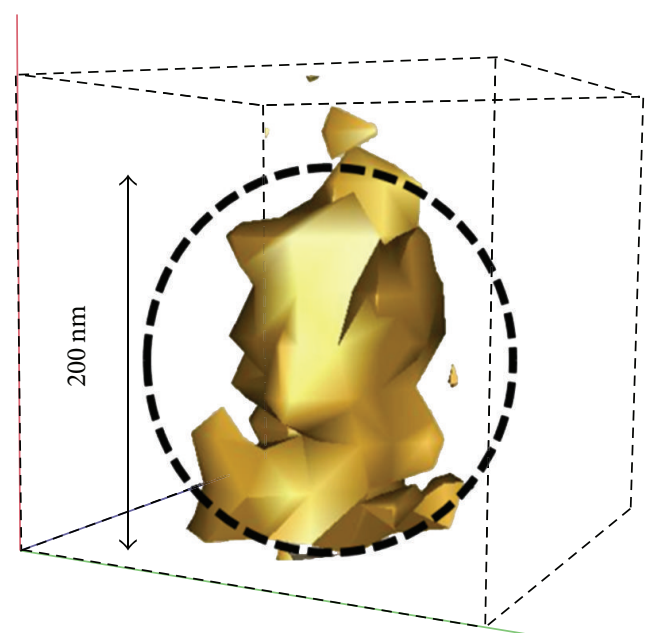

(b)

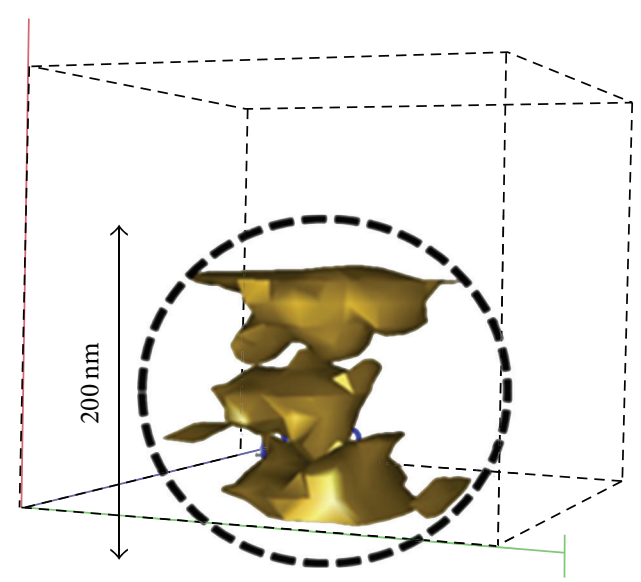

(d)

FIGURE 5: Three-dimensional images of a single nanoparticle: (a) surface image with general nonlinear confocal microscope $\left(I=1.0 \mathrm{~W} / \mathrm{cm}^{2}\right)$, (b) inspection image with polarization interferometric linear confocal microscope $\left(I=8.0 \mathrm{~W} / \mathrm{cm}^{2}\right)$, and (c)-(d) inspection images with polarization interferometric nonlinear confocal microscope $\left(I=1.0 \times 10^{2} \mathrm{~W} / \mathrm{cm}^{2}\right.$ and $\left.I=2.5 \times 10^{3} \mathrm{~W} / \mathrm{cm}^{2}\right)$. 


\section{Conflict of Interests}

The authors declare that there is no conflict of interests regarding the publication of this paper.

\section{Acknowledgment}

This work has been supported in part by a grant-in-aid for fundamental scientific research from the Ministry of Education, Culture, Sports, Science and Technology of Japan.

\section{References}

[1] R. Barillé, P. Tajalli, S. Kucharski, E. Ortyl, and J.-M. Nunzi, "Photoinduced deformation of azopolymer nanometric spheres," Applied Physics Letters, vol. 96, no. 16, Article ID 163104, 2010.

[2] W. Weise, P. Zinin, T. Wilson, A. Briggs, and S. Boseck, "Imaging of spheres with the confocal scanning optical microscope," Optics Letters, vol. 21, no. 22, pp. 1800-1802, 1996.

[3] X. Yu, Y. Araki, K. Iwami, and N. Umeda, "Measurement of nanoparticle sizes by conventional optical microscopy with standing evanescent field illumination," Optics Letters, vol. 33, no. 23, pp. 2794-2796, 2008.

[4] C. Egami, N. Nishimura, and T. Okawa, "Jitter-free multi-layered nanoparticles optical storage disk with buffer ring," Optics Express, vol. 18, no. 15, pp. 15901-15906, 2010.

[5] W. H. de Jong and P. J. A. Borm, "Drug delivery and nanoparticles: applications and hazards," International Journal of $\mathrm{Na}$ nomedicine, vol. 3, no. 2, pp. 133-149, 2008.

[6] S. Chowdhury, A. Dhalla, and J. Izatt, "Structured oblique illumination microscopy for enhanced resolution imaging of nonfluorescent, coherently scattering samples," Biomedical Optics Express, vol. 3, no. 8, pp. 1841-1854, 2012.

[7] V. K. Gupta and J. A. Kornfield, "Polarization modulation laser scanning microscopy: a powerful tool to image molecular orientation and order," Review of Scientific Instruments, vol. 65, no. 9, pp. 2823-2828, 1994.

[8] V. I. Shcheslavskiy, S. M. Saltiel, A. R. Faustov, G. I. Petrov, and V. V. Yakovlev, "How to measure $\chi(3)$ of a nanoparticle," Optics Letters, vol. 31, no. 10, pp. 1486-1488, 2006. 

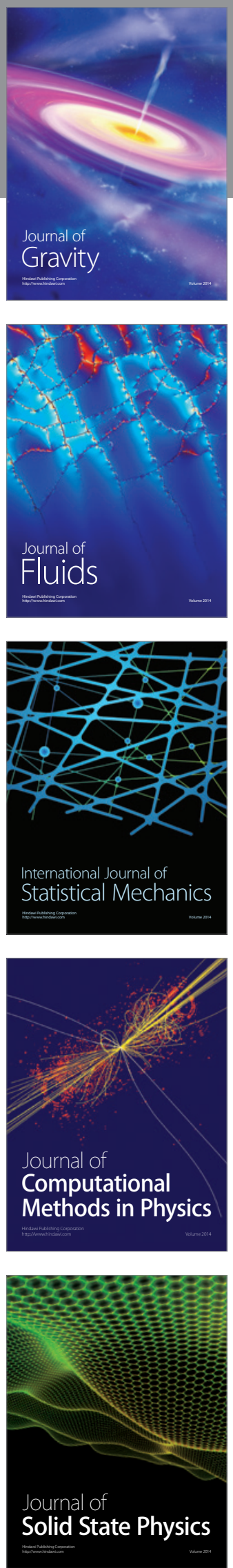

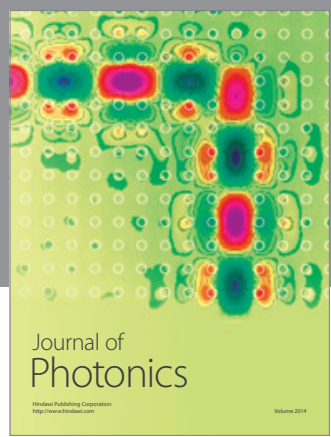

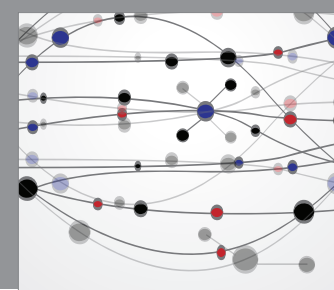

The Scientific World Journal

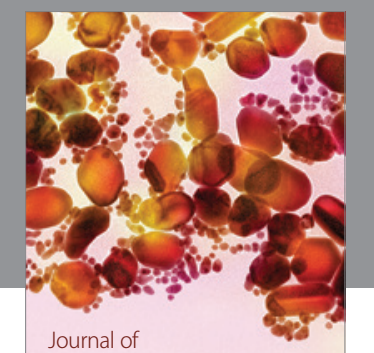

Soft Matter
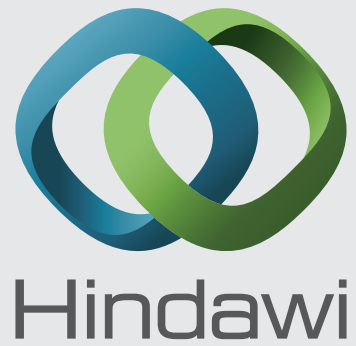

Submit your manuscripts at

http://www.hindawi.com
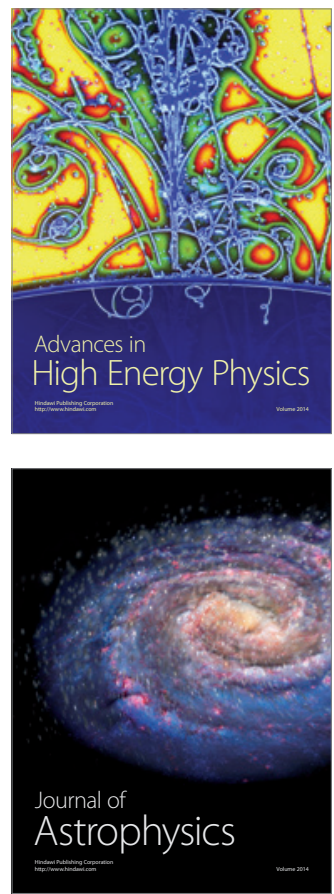
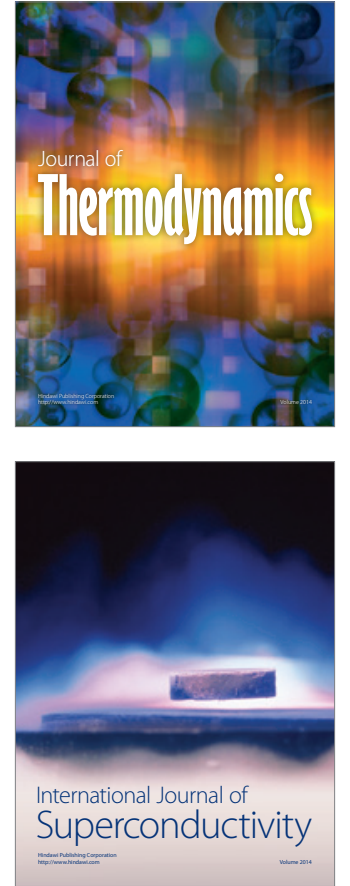
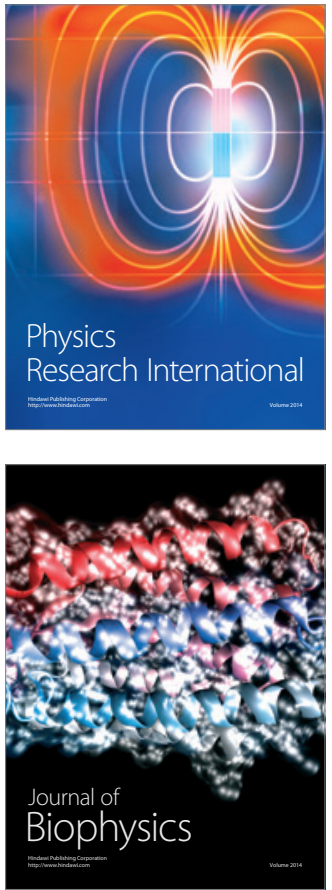
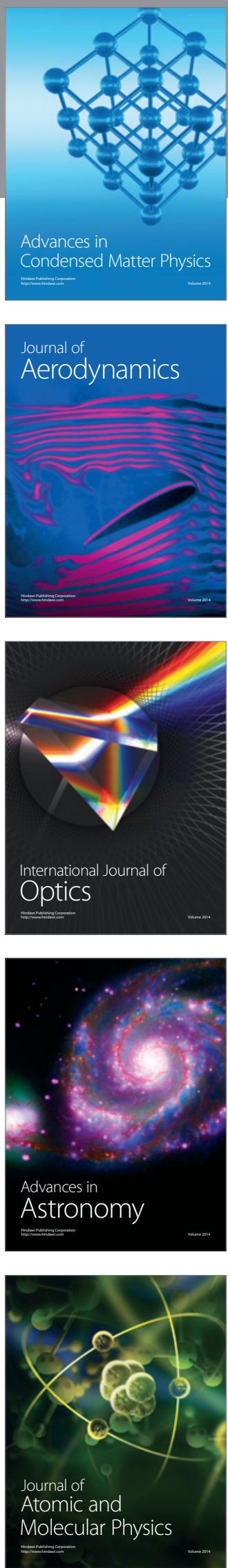